\title{
Assessment of a TEC calibration procedure by single-frequency PPP
}

\author{
Fabricio dos Santos Prol ${ }^{1}$. Paulo de Oliveira Camargo ${ }^{1}$. João Francisco Galera Monico ${ }^{1}$. \\ Marcio Tadeu de Assis Honorato Muella ${ }^{2}$
}

Received: 25 April 2017 / Accepted: 10 January 2018 / Published online: 18 January 2018

(c) Springer-Verlag GmbH Germany, part of Springer Nature 2018

\begin{abstract}
Global navigation satellite system (GNSS) measurements have become an outstanding data source for ionospheric studies using total electron content (TEC) estimation procedures. Many methods for TEC estimation had been developed over recent decades, but none of them is capable of providing high accuracy for the single-frequency precise point positioning (PPP). We present an analysis of the performance of a new TEC calibration procedure when applied to PPP. TEC estimation is assessed by calculating the improvements obtained in single-frequency PPP in kinematic mode. A total of 120 days with six distinct configurations of base and rover stations was used, and the TEC performance is assessed by applying the estimated TEC from the base station to correct the ionospheric delay in a nearby rover receiver. The single-frequency PPP solution in the rover station reached centimeter accuracy similar to the ionospheric-free PPP solution. Further, the TEC calibration method presented an improvement of about $74 \%$ compared to the PPP using the global ionospheric maps. We, therefore, confirm that it is possible to estimate high-precision TEC for accurate PPP applications, which enables us to conclude that the principal challenge of the GNSS community developing ionospheric models is not the differential code bias or the temporal variation of the ionosphere, but the development of methods for accurate spatial interpolation of the slant TEC.
\end{abstract}

Keywords GIM · Kinematic PPP · DCB · GPS · Ionospheric delay

\section{Introduction}

Total electron content (TEC) is one of the most important parameters used to describe the proprieties of the ionosphere. With the aim of describing TEC in space and time, ionospheric models use the dispersive propriety of the ionosphere on global navigation satellite system (GNSS)

Fabricio dos Santos Prol

fabricioprol@hotmail.com

Paulo de Oliveira Camargo

paulo@fct.unesp.br

João Francisco Galera Monico

galera@fct.unesp.br

Marcio Tadeu de Assis Honorato Muella mmuella@univap.br

1 Universidade Estadual Paulista - UNESP, Roberto Simonsen, 305, Presidente Prudente, São Paulo 19060-900, Brazil

2 Laboratório de Física e Astronomia, IP\&D, Universidade do Vale do Paraíba - UNIVAP, São José dos Campos, São Paulo 12244-000, Brazil signals to describe the ionosphere in global, regional and local scales. As a result of having ionospheric models, several applications can be performed, such GNSS positioning (Macalalad et al. 2016), morphology and dynamics of the ionosphere (Lin et al. 2005; Biqiang et al. 2007), and ionospheric monitoring. However, when considering high accurate determinations, the TEC estimation procedures still present an ongoing challenge to the scientific community.

Three of the main facts that limit the accuracy of TEC estimated using ionospheric models based on GNSS data are ionospheric variability using spatial/temporal interpolations, intrinsic errors of the mapping functions for converting TEC in vertical TEC (VTEC) and differential instrumental biases between the GNSS observations in L band (Coco et al. 1991; Sardón and Zarraoa 1997; Li et al. 2012; Jin et al. 2016). Although the GNSS networks provide dense information about the ionosphere, there is still a considerable computational limitation and lack of data for interpolating TEC data with high accuracy, mainly in zones with high electron density variability. In this regard, approximations are generally used by projecting VTEC to make the spatial/temporal interpolation easier. Also, the differential bias includes a rank 
deficiency in the equation system of ionospheric models, and it is consequently necessary to include constraints in the TEC estimations (Camargo et al. 2000). Global ionospheric maps (GIMs) generated by the International GNSS Service (IGS), for example, are produced by imposing the sum of the differential code bias (DCBs) of the satellites to be equal to zero (Montenbruck et al. 2014) or using exclusively carrier phase data, where the instrumental delay is estimated together with the ambiguities (Hernández-Pajares et al. 1999). These approaches are efficient for obtaining leastsquare solution with minimum residuals. However, they do not describe any physical characteristics of the instrumental biases proprieties. For this reason, there is no concrete knowledge about the absolute accuracy of the instrumental biases. Hence, we do not know the absolute accuracy of TEC for many ionospheric models.

The precision of TEC can be assessed by calculating the improvement it provides when performing GNSS positioning with single-frequency receivers. Many researchers have already used such receivers and ionospheric corrections estimated by GIMs to obtain, in general, an absolute accuracy of $0.5 \mathrm{~m}$ in the horizontal and $1 \mathrm{~m}$ in vertical for precise point positioning (PPP) in kinematic mode ( $\varnothing$-vstedal 2002; Le and Tiberius 2007; Sterle et al. 2015). However, the accuracy of these results is significantly worse than those provided by dual-frequency PPP, since dual-frequency PPP enables the elimination of first-order ionospheric effects, leading to sub-centimeter accuracy (He et al. 2014). Since the single-frequency PPP using GIM presents a metric solution, we may conclude that the procedure of TEC estimation is not satisfactory for higher accuracy applications. Therefore, there is still room for improvement.

Instead of using global or regional ionospheric models, TEC calibration procedures can be utilized for the estimation of ionospheric delay. Many methods have already been proposed to perform the TEC calibration (Ciraolo et al. 2007; Arikan et al. 2008; Montenbruck et al. 2014; Prol and Camargo 2014). However, the accuracy of single-frequency PPP using TEC estimated with such procedure remains unknown. Therefore, an issue that still concerns the scientific community is whether the developed TEC calibration procedures are good enough to provide accurate results in PPP.

With the objective of showing the performance of TEC calibration procedures for PPP applications, we present a new procedure to estimate TEC based on GIM and evaluate its performance by analyzing the improvement to singlefrequency PPP in kinematic mode. The TEC is estimated using dual-frequency GNSS stations and directly applied in nearby stations. Using this strategy, we were able to obtain an accurate solution in the PPP results. The single-frequency PPP accuracy obtained is similar to that from dual-frequency PPP, making it possible to affirm that the procedure is capable of providing TEC for accurate PPP applications.
Although the relative positioning could be used to provide a better GNSS solution for nearby stations, the main goal of the presented discussion was not to provide a new method to perform the GNSS positioning, but to show that the currently used calibration procedures are capable of providing high-precision TEC. Indeed, PPP is only used to validate the performance of the TEC estimation procedure.

Many GNSS applications can benefit from highly precise TEC, such as for input to ionospheric models based on GNSS data, calculating ionospheric gradients to be included in ionospheric threat models for GNSS argumentation systems (Datta-Barua et al. 2010), and for improving time convergence of ambiguities in PPP solutions using L1 and L2 linked with initial slant ionospheric delays (Li et al. 2013). The next section, therefore, presents the proposed method to estimate TEC, the following section shows the results and, in the end, we present a discussion and conclusions about the results and also about limitations of the ionospheric models currently being used for single-frequency PPP.

\section{TEC estimation procedure}

Early estimation procedures to derive TEC from GNSS observations in conjunction with ionospheric models have been developed by Georgiadiou (1994) and Wild (1994), demonstrating that these were efficient in determining ionospheric delay for some applications using single-frequency receivers. Once GPS constellation became operational, the IGS established the IGS Ionosphere Working Group (IGS IonoWG) in 1998 with the main goal of continuous ionospheric monitoring (Hernández-Pajares et al. 2009). Therefore, a global network of GPS stations with dual-frequency receivers is used for producing ionospheric models (Feltens 1998; Mannucci et al. 1998; Hernández-Pajares et al. 1999; Schaer 1999) and the results were made available in the form of GIMs. Simultaneously, regional models were developed, such as Modion (Camargo et al. 2000) and the La Plata Ionospheric Model (Brunini et al. 2004).

Today, several approaches can be used for ionospheric modeling, such as polynomial models (Azpilicueta et al. 2006; Alizadeh et al. 2015), splines (Durmaz and Karslioglu 2015), grid-based methods (Otsuka et al. 2002), tomographic algorithms (Mitchell and Spencer 2003; Wen et al. 2012; Prol and Camargo 2016), ingestion data processes (Migoya-Orué et al. 2015) or data assimilation methods (Schunk et al. 2004; Hajj et al. 2004; Bust and Mitchell 2008). A similar characteristic of these methods is that, before using TEC for GNSS positioning, the TEC is described by ionospheric models or calculated through grid-based processes that use interpolations, iterative reconstruction methods or least-square adjustment procedures. However, due to high ionospheric variability, computational limitations, and the spatial and temporal resolution of the 
ionospheric grids, these algorithms have presented significantly worse results in GNSS positioning than ionosphericfree solutions.

An alternative way of obtaining TEC that also requires less computational effort than ionospheric modeling is performing TEC calibration procedures. Some studies have used calibration procedures for TEC estimation (Ciraolo et al. 2007; Arikan et al. 2008; Montenbruck et al. 2014; Prol and Camargo 2014). However, the accuracy of single-frequency PPP using TEC estimated with such procedure remains unknown. In this context, this section shows the new procedure for TEC calibration that provides a significant improvement in singlefrequency GNSS positioning.

Our method is implemented in two steps. The first one estimates ambiguities and DCBs. The second estimates TEC. Ambiguities are estimated using so-called phase leveling, which is based on code information (Mannucci et al. 1998; Ciraolo et al. 2007). In phase leveling, the difference between ambiguity terms is determined by the mean difference of the ionospheric delay calculated using phase and code over one arc of data with no cycle slips. Thus, the leveling ambiguity $(\Delta N)$ in a unique arc of continuous data is calculated through:

$\Delta N=\left(\lambda_{1} N_{1}-\lambda_{2} N_{2}\right)=\frac{1}{n_{\text {obs }}} \sum_{j=1}^{n_{\text {obs }}}\left[\left(P_{2 j}-P_{1 j}\right)-\left(\lambda_{1} \phi_{1 j}-\lambda_{2} \phi_{2 j}\right)\right]$

where $\lambda$ represents the wavelength $(\mathrm{m})$ of the carrier in L1 and L2, $\phi$ is the carrier phase observation of GPS (cycles), $P$ is the pseudorange $(\mathrm{m}), N$ is the ambiguity (in cycles), $n_{\mathrm{obs}}$ is the number of observations in the arc with continuous data. Only arcs with a minimum of 5 min of continuous data were used in the experiments.

Once the leveling ambiguities are calculated for all the continuous arcs of a specific receiver station and specific day, satellite DCBs are directly obtained from the IGS solutions organized in IONosphere map EXchange format (IONEX) files. The receiver DCB is given by the weighted mean of the difference of phase difference $\left(\lambda_{1} \phi_{1 j}-\lambda_{2} \phi_{2 j}\right)$ and the calculated TEC, the leveling ambiguity, and the satellite DCB, as:

$$
\begin{aligned}
c \Delta b_{\mathrm{r}}= & \frac{1}{\sum_{i=1}^{n_{\mathrm{arc}}} \sum_{j=1}^{n_{\mathrm{obs}}} w_{i j}} \sum_{i=1}^{n_{\text {arc }}} \sum_{j=1}^{n_{\mathrm{obs}}} w_{i j} \\
& \times\left[\left(\lambda_{1} \phi_{1 i j}-\lambda_{2} \phi_{2 i j}\right)-\frac{\mathrm{TEC}_{i j}^{\mathrm{inx}}}{F}-\Delta N_{i}-c \Delta b_{\mathrm{s} i}\right]
\end{aligned}
$$

with:

$F=\frac{f_{1}^{2} f_{2}^{2}}{40.3\left(f_{1}^{2}-f_{2}^{2}\right)}$

where $f$ is the frequency $(\mathrm{Hz})$ of the carrier, $\Delta b_{\mathrm{s}}$ and $\Delta b_{\mathrm{r}}$ are the DCBs (seconds) of the satellite and receiver, $c$ is the speed of light in vacuum $\left(\mathrm{m} / \mathrm{s}^{2}\right), \mathrm{TEC}_{i j}^{\mathrm{inx}}$ is TEC derived from IONEX files, $w$ is the weighting parameter for each $\mathrm{TEC}_{i j}^{\mathrm{inx}}$ and $n_{\text {arc }}$ expresses the number of arcs with continuous data for 1 day of observation. The receiver DCB is thereby defined with a unique value for a whole day. Also, the weighting parameter is defined as:

$w_{i j}=\frac{1}{\left(\sigma_{\mathrm{TEC}_{\mathrm{ij}}^{\mathrm{inx}}} / F\right)^{2}}$

where $\sigma_{\mathrm{TEC}_{i j}^{\mathrm{inx}}}$ is the standard deviation of TEC derived from the root-mean-square maps available in IGS IONEX files.

To retrieve TEC $_{i j}^{\text {inx }}$ from IGS VTEC maps, the TEC is projected in a point located in a single layer with constant height. The projection point, called ionospheric pierce point (IPP), is represented at the intersection of the satellite to receiver vector with a single layer located at $450 \mathrm{~km}$ above the earth's surface. A bilinear interpolation in space and time on VTEC maps is done to obtain VTEC at the IPP location. The VTEC is then converted to TEC using a mapping function, where the standard geometric mapping function is given by:

$\mathrm{TEC}^{\mathrm{inx}}=\frac{\mathrm{VTEC}_{\mathrm{IPP}}}{\cos \left(z^{\prime}\right)}$

with:

$\sin \left(z^{\prime}\right)=\frac{r_{\mathrm{m}}}{r_{\mathrm{m}}+h_{\mathrm{m}}} \sin (z)$

where $z$ is the zenithal angle projected in the path of the GPS signal, $r_{\mathrm{m}}$ is the mean earth radius and $h_{\mathrm{m}}$ is the height of the single layer.

As the satellites DCB and TEC are obtained from the IGS VTEC maps, it is expected that the estimated receiver DCB will have similar values as the receiver DCB available in the IONEX files for all the GNSS stations that were used to generate the IGS VTEC maps. In addition, the DCB estimated for stations that were not used to produce the IGS VTEC maps will be related to the DCB reference frame defined by IGS. Therefore, the proposed method is used to estimate the receiver DCB in the same DCB reference frame defined by IGS. A similar approach was used in previous works, such as in Arikan et al. (2008), with the main difference concerning the incorporation of the weighting parameter. However, despite this being a new method, the differences are not sufficiently different in the sense that the main goal of this paper is not about a new method, but to show the performance of TEC obtained from such methods when applying the calibrated TEC in PPP.

Once the leveling ambiguities and DCBs are obtained, TEC is directly calculated along the path of the GPS signal 
from the satellite to receiver in a single epoch using the following equation:

$\mathrm{TEC}=F\left[\left(\lambda_{1} \phi_{1}-\lambda_{2} \phi_{2}\right)-\Delta N-c\left(\Delta b_{\mathrm{s}}+\Delta b_{\mathrm{r}}\right)\right]$

where the solution of (7) is obtained from the determination of the difference of the ambiguous terms $\left(\lambda_{1} N_{1}-\lambda_{2} N_{2}\right)$ and DCBs in the first step.

GNSS stations were defined in pairs in order to estimate TEC in one of them by (7), which is referred to as the base, and use the estimated TEC to mitigate the ionospheric delay in a nearby station, called rover. Ignoring the high-order terms of the ionosphere (Elmas et al. 2011; Marques et al. 2011), the ionospheric delay is calculated by the following equation:

$I_{L_{i}}=\frac{40.3}{f_{L_{i}}^{2}} \mathrm{TEC}$

where $I$ is the ionospheric delay to be used for mitigating the ionospheric refraction at the rover receiver. It is interesting to notice that the errors of the mapping function are avoided when using such procedure.

The main problems with this approach are the distance between stations, failures in data recording and constant signal failures. The distance between the stations were defined with a maximum of $35 \mathrm{~km}$ in order to minimize the problems of the spatial variability of the ionosphere and focus the analysis on the possibility of obtaining a similar precision than the ionospheric-free solution. However, it is important to note that significant errors in the PPP solution will be obtained if the temporal variations of TEC are not well estimated. Since the estimated TEC is related to the reference frame of the IGS DCBs, the calibrated TEC is very close to the TEC from IGS VTEC maps. Therefore, at instants without data from the base station, it is possible to fill the temporal TEC gaps using TEC retrieved from GIMs. The TEC retrieved through the IGS VTEC maps for instants without data in base station is then used in the rover station to correct the ionospheric delay in PPP without including significant variations in the temporal description of TEC. In fact, DCBs were estimated using GIMs, and then, the improved TEC was estimated using such DCBs with the main reason to fill the temporal TEC gaps in the rover receiver at instants without data in the base station.

It is worth mentioning that when using PPP for evaluating TEC performance, the errors in the DCB estimation will be absorbed by the PPP receiver clock unknown. The bias in the absolute level of TEC is mostly absorbed by the receiver clock, so the absolute accuracy of TEC remains unknown. Therefore, the intention is to verify whether the proposed TEC estimation procedure is capable of providing temporal variation of TEC with sufficient precision to be used in PPP applications. Even considering an absolute error in the TEC calibration, we can verify whether the temporal variability of the estimated TEC is good.

\section{Investigation of the TEC performance in single-frequency PPP}

PPP at distinct stations was performed for evaluation of the estimated TEC. Kinematic processing mode was carried out to analyze the TEC performance for each epoch in which observations were available. The location of stations is presented in Fig. 1. Such a selection enabled us to analyze TEC for distinct configurations of processing, where GNSS data of the stations are freely available from the IGS network and the Rede Brasileira de Monitoramento Contínuo (RBMC) network.

Table 1 shows the types of receivers and antennas deployed at the stations, the distance between the stations and the identification of the stations used to calculate TEC and to process PPP. We chose pairs a relatively short

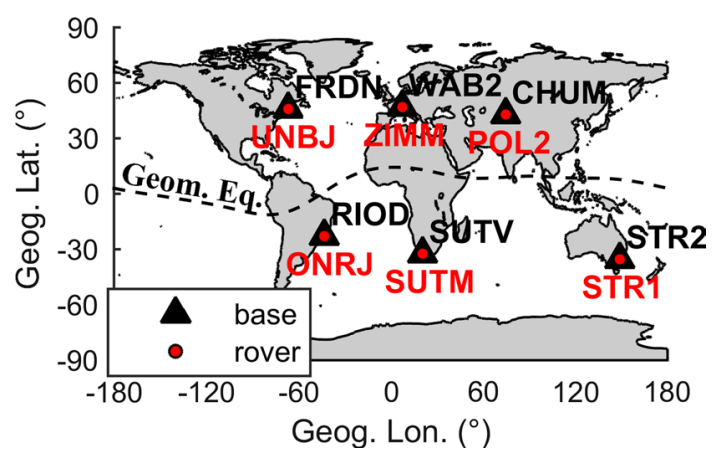

Fig. 1 Stations used for TEC estimation and PPP evaluation. The geomagnetic equator is represented by the dashed line

Table 1 Instruments and distances $(\mathrm{km})$ between rover and base

\begin{tabular}{llll}
\hline Stations & Receiver & Antenna & Distance $(\mathrm{km})$ \\
\hline STR2 & Trimble NetR8 & TRM59800.00 & - \\
STR1 & Leica GRX1200 & ASH701945C_M & 0.07 \\
SUTV & JPS EGGDT & ASH701945G_M & - \\
SUTM & AOA benchmark & AOAD/M_T & 0.14 \\
FRDN & TPS NetG3 & TPSCR.G3 & - \\
UNBJ & TPS legacy & TRM57971.00 & 2.33 \\
WAB2 & Ashtech Z-XII3T & ASH700936F_C & - \\
ZIMM & Trimble NetRS & TRM29659.00 & 5.20 \\
RIOD & Trimble NetRS & TRM41249.00 & - \\
ONRJ & Leica GRX1200 & LEIAX1203 & 12.04 \\
CHUM & Trimble NetRS & AOAD/M_T & - \\
POL2 & Ashtech UZ-12 & TPSCR.G3 & 35.73 \\
\hline
\end{tabular}


distance apart, and we selected stations with receivers and antennas from different brands in order to avoid possible correlations between clock and ambiguities obtained in the TEC estimation procedure.

RTKLIB (Takasu and Yasuda 2009) was used to perform PPP. Some adaptations were implemented in order to use the estimated TEC. In the experiments, three configurations of PPP were analyzed: (a) using the ionospheric-free observation (PPP/if); (b) using L1 and ionospheric delay correction from IONEX files produced by Center for Orbit Determination in Europe (CODE) to define the single-frequency PPP solution with a traditional ionospheric model (PPP/inx); and (c) using L1 and TEC estimated through the proposed method (PPP/tec). The standard deviation of TEC on PPP/ tec was defined empirically as thirty times more precise than the TEC from IONEX files.

Among the PPP configurations using RTKLIB software, we used the following: (1) combined solution obtained by forward and backward filters; (2) cutoff angle of $10^{\circ}$; (3) earth tides corrections; (4) estimation of tropospheric delay during PPP; (5) precise ephemerides (sp3) and satellite clock corrections (clk_30s) acquired from IGS products; (6) daily Receiver Independent Exchange Format (RINEX) files with 30-s interval for data collection; (7) only GPS constellation; (8) correction of the Phase Center Variation (PCV) of antenna obtained through IGS products; (9) phase windup corrections; (10) no strategy for ambiguity solution; and (11) corrections of the differential instrumental bias between the civil and precise codes (C1-P1) when P1 was not available.

GPS data for a total of 120 days in the year 2013 were processed and analyzed for each of the six pairs of stations: 60 days refer to the beginning of the year (January and February) when the solar position of summer solstice was located in the southern hemisphere, and 60 days refer to July and August when the summer Sun was located in the northern hemisphere. These days were selected in order to evaluate the method in an epoch with high ionospheric variability, but with no intense scintillations that could affect PPP. The reference coordinates of the IGS stations were obtained from the International Terrestrial Reference Frame 2014 (ITRF2014), epoch 2010, and the reference coordinates of RBMC stations were obtained from SIstema de Referencia Geocéntrico para las AméricaS (SIRGAS) solutions at epoch 2013. A time update was performed on the reference coordinates to make them consistent with the PPP solutions.

Two main analyses were performed to evaluate TEC: The first was carried out to analyze the estimation procedure of TEC and DCB for the base stations, and the second was conducted to evaluate the improvement the estimated TEC offers to PPP at the rover stations. The next section presents the results of the estimated TEC in the base stations in order to give an overview of the main differences of the estimated TEC and DCB in comparison with TEC and DCB obtained through IONEX files. Then, in the following section, we show the PPP results when applying the proposed method. Two parameters were used to evaluate three-dimensional (3D) PPP accuracy: the standard deviation and the rootmean-square error (RMSE) of the solutions.

\section{TEC and DCB estimation}

Figure 2 (top) shows an example of day 46 of 2013 of the TEC discrepancies calculated for each satellite at station CHUM when using the proposed method and IONEX files. The values are expressed in TEC Units (TECU- $10^{16} \mathrm{el} /$ $\mathrm{m}^{2}$ ), where it can be seen that the discrepancies vary between - 20 and 20 TECU. Also, a significant difference was obtained in the temporal variation of TEC, which can be observed in the example showed in bottom panel. The temporal variation of TEC-CODE (black lines) is smoothed due to the linear interpolation performed to obtain VTEC in the IPP points at each instant. However, for the proposed method, TEC was represented with higher temporal variability since it is estimated using GNSS observations at every epoch.

While we present details of TEC results for a single station and day, the behavior of the discrepancies between the estimated TEC and TEC-CODE was similar for all other stations and days. A summary of the discrepancies for all stations and the 120 days is listed in Table 2. We present the mean of the discrepancies, the root-mean-square difference (RMSD), the maximums (Max) and the minimums (Min). In general, it can be seen that the mean discrepancy tends to zero, which means
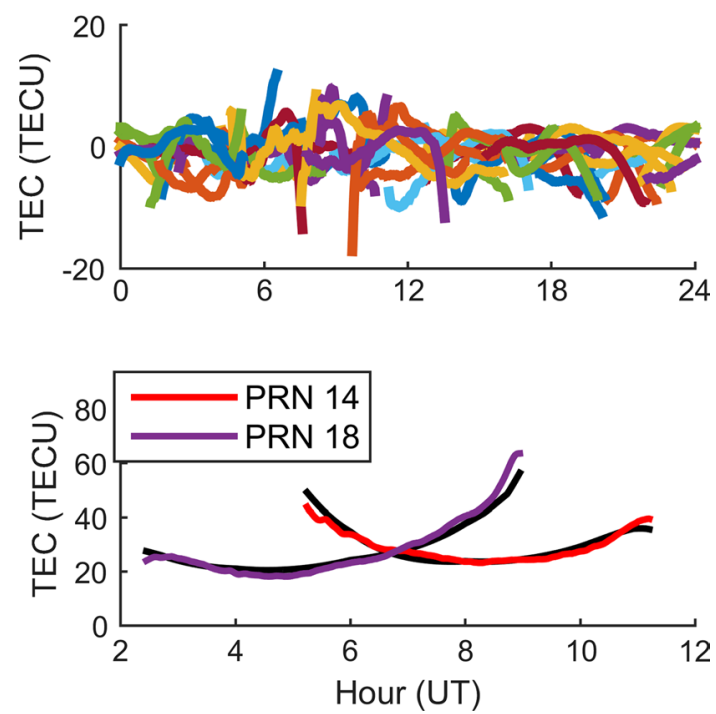

Fig. 2 TEC discrepancies (top) between the proposed method and TEC-CODE for day 46 of 2013 at CHUM station and a visual comparison of TEC (bottom) for GPS PRNs 14 and 18. Black lines in the bottom panel are the TEC-CODE values 
Table 2 Statistical comparison of the discrepancies between the estimated TEC and TEC-CODE

\begin{tabular}{lllll}
\hline Base stations & Mean & RMSD & Max & Min \\
\hline STR2 & -0.02 & 1.44 & 17.19 & -21.06 \\
SUTV & -0.17 & 1.54 & 19.53 & -26.03 \\
FRDN & -0.25 & 1.39 & 31.52 & -34.74 \\
WAB2 & -0.39 & 1.46 & 16.99 & -23.22 \\
RIOD & -0.23 & 2.06 & 51.55 & -39.83 \\
CHUM & -0.53 & 1.83 & 38.36 & -37.47 \\
Mean of all & -0.27 & 1.62 & 29.19 & -30.42 \\
\hline
\end{tabular}

The unit of the values is TECU

that there is no systematic difference between the methods for representing the daily behavior of TEC. Analyzing the other statistics, it can be verified that the temporal variations of TEC considering every $30 \mathrm{~s}$ has a mean dispersion of 1.62 TECU, with maximums and minimums of 29.19 TECU and - 30.42 TECU, respectively. Also, based on Table 2, differences between ionospheric delay to be used in PPP/inx and $\mathrm{PPP} /$ tec can be calculated. Since 1 TECU provides an error of approximately $16 \mathrm{~cm}$ in the ionospheric delay to be used in single-frequency PPP, we can see that the RMSD is about $26 \mathrm{~cm}$ for the ionospheric delay discrepancy.

An analysis of the differences between the estimated DCBs from the receivers and the receiver DCBs of CODE is presented in Fig. 3. Just a few stations are presented because they were the only base stations used to produce the IONEX files. In general, the means of the discrepancies are $0.14,0.07,0.07$ and $0.12 \mathrm{~ns}$ for STR2, FRDN, SUTV and WAB2, respectively. Therefore, it can be seen that the DCB discrepancies have low
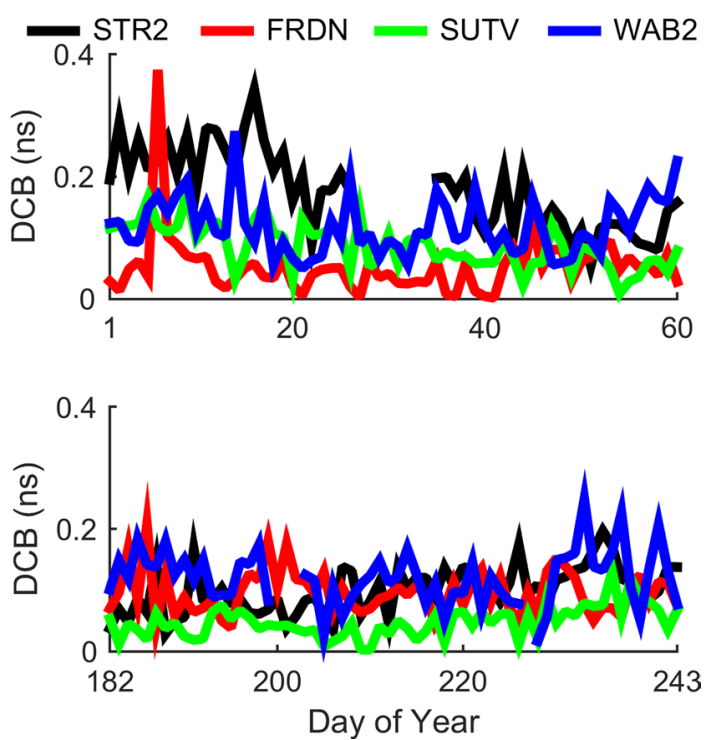

Fig. 3 Discrepancies between the DCB obtained from IONEX and estimated with the proposed method when the solar position was located in the southern hemisphere (top) and when the summer sun was located in the northern hemisphere (bottom) variability over consecutive days, and the results are similar to the CODE DCBs. In addition, one can see that the DCB discrepancies followed the ionospheric variability. Larger discrepancies in SUTV and STR2 were calculated at the beginning of the year (days 1-60), when the summer solstice sun was located in the southern hemisphere, and lower discrepancies were obtained between days 182 and 243, when the summer solstice sun was located in the northern hemisphere. On the other hand, stations WAB2 and FRDN presented an inverse behavior, showing larger discrepancies between 182 and 243 because they are located in the northern hemisphere.

\section{Assessment of TEC by single-frequency PPP}

In a preliminary evaluation, we analyzed the results in a single day to show in detail the behavior of PPP results when applying the estimated TEC for mitigating the ionospheric delay in the rover stations. Day 46 of 2013 was selected because it showed a representative behavior in comparison with other days. Figure 4 presents the 3D error calculated for each epoch for PPP in the kinematic mode, where it is possible to see a significant improvement of PPP/tec in comparison with PPP/ inx and a similar centimeter accuracy as compared to PPP/if for several epochs. The mean error was equal to $0.51,0.09$ and $0.03 \mathrm{~m}$ for PPP/inx, PPP/tec and PPP/if, respectively.

The solution of PPP/tec was worse than PPP/if at a level of centimeters in some epochs, as can be seen for the station
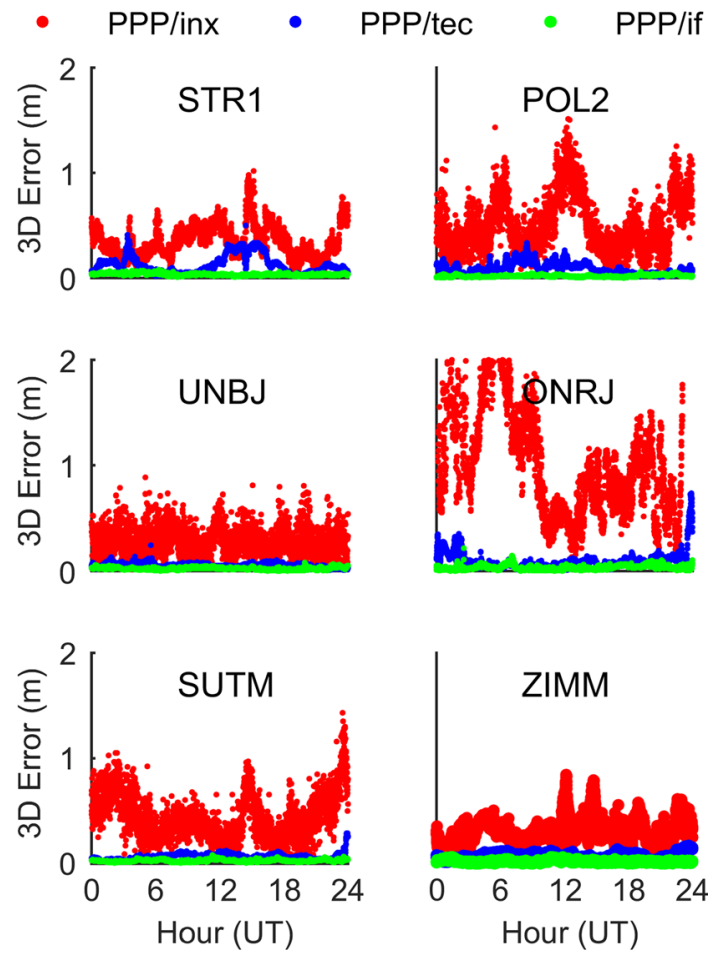

Fig. 4 3D error for day 46 of 2013 
POL2, between 04 and $12 \mathrm{~h} \mathrm{UT}$, and station STR1, at $04 \mathrm{~h}$ UT and between 12 and $16 \mathrm{~h} \mathrm{UT}$. This centimeter discrepancy in POL2 is justified due to the distinct ionospheric conditions that affected the base station CHUM, which is located $35.73 \mathrm{~km}$ away from the rover station. When estimating TEC in CHUM and POL2 using the proposed method, Fig. 5-bottom panel, we verify that the magnitude of the discrepancy between TEC reaches up to 3-5 TECU for some satellites in the interval between 04 and $12 \mathrm{~h} \mathrm{UT}$. This magnitude is similar to the magnitude of the TEC discrepancies in other periods, or even in comparison with the TEC differences between STR1 and STR2. However, during 04-12 h UT we can see high variability for the temporal behavior of the TEC difference, which allows us to be sure that the major fact that degraded PPP/tec was due to problems in representing the temporal variations of TEC in POL2. For STR1, the PPP/tec degradation was not due to the distances between base and rover, as shown in Fig. 5 (top), where there is low variability of the TEC difference between STR1 and STR2. Actually, the main problem is due to registration failures in data acquisition. When there are no observational data, the PPP/tec algorithm calculates TEC using the IONEX files, which justify the similar performance of PPP/tec and PPP/inx shown in Fig. 4.

The same PPP configuration presented in Fig. 4 is used to generate Fig. 6, but now showing the standard deviation of the solution. Despite problems estimating TEC to be used in rover stations, it is quite clear that the PPP/tec showed a similar precision of the solution compared to PPP/if. Also, the standard deviation of PPP/tec was systematically better
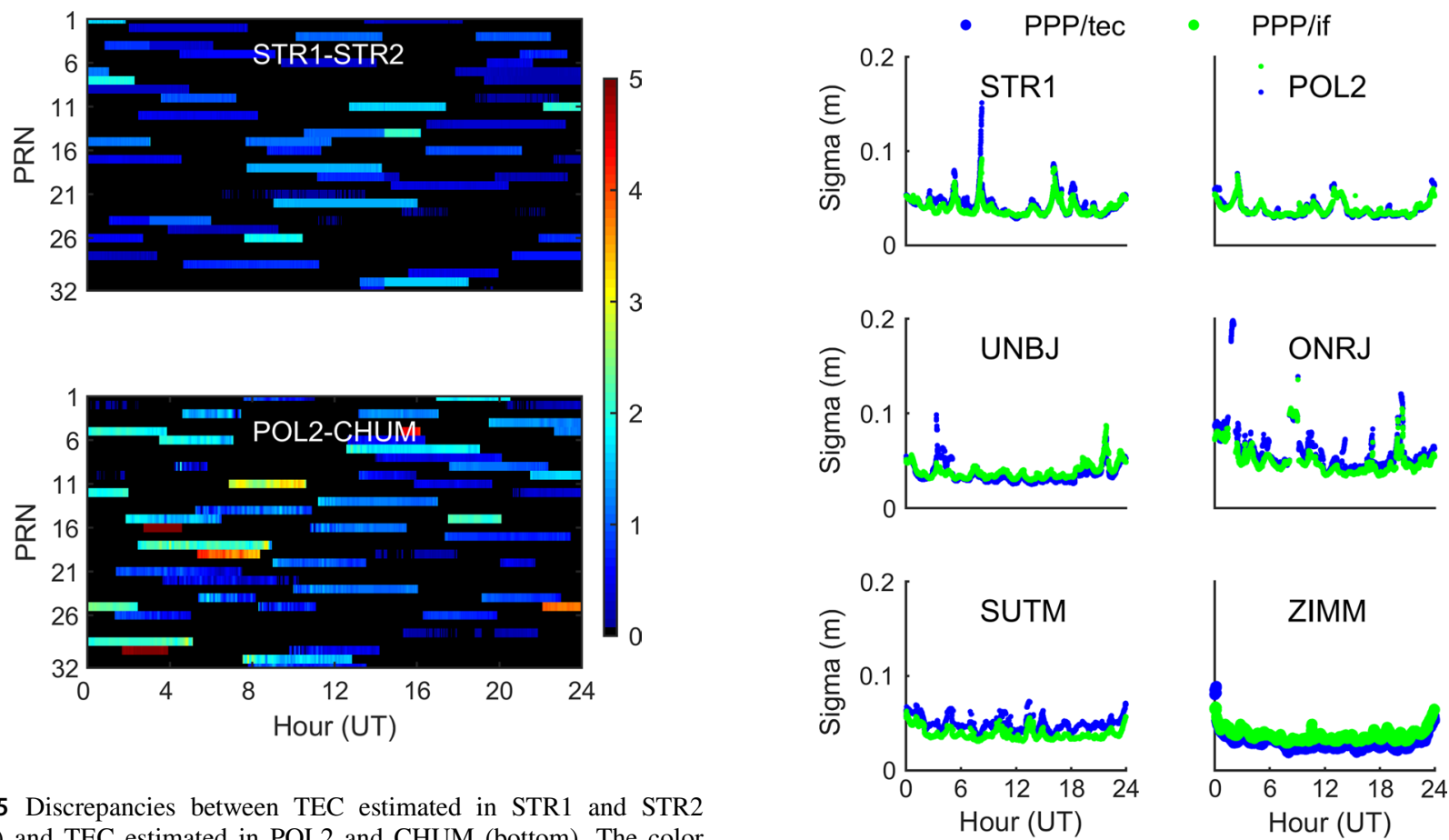

Fig. 5 Discrepancies between TEC estimated in STR1 and STR2
(top) and TEC estimated in POL2 and CHUM (bottom). The color bar represents the discrepancy in TECU, where the black color represents the absence of data

than the standard deviation of PPP/if for UNBJ and ZIMM, which could be due to the noise of the ionospheric-free observations. In general, the mean standard deviation of day 46 was $0.66,0.05$ and $0.05 \mathrm{~m}$ for PPP/inx, PPP/tec and PPP/ if, respectively, and the standard deviation of IONEX is not shown due to their high values.

Despite showing details only for day 46 , the daily behavior analysis of PPP/tec can be extended to other days, except for ONRJ, which is located on the southern crest of the Equatorial Ionization Anomaly (EIA). For a complete characterization of PPP/tec performance in ONRJ, we present typical results of the worst performance of PPP/tec in Fig. 7. We show the 3D errors calculated for every PPP epochs and the differences between TEC estimated for ONRJ and RIOD. For all three cases shown, day 5 represents the most common result regarding the worst performance of PPP/tec. The initial hours of day 5 (00-04 h UT) correspond to the period when enhanced TEC structures, associated with the wellknown evening prereversal enhancement of plasma drift in the equatorial low-latitude ionosphere, are expected to affect the region of stations ONRJ and RIOD. The distance between the ONRJ and RIOD stations is about $12.04 \mathrm{~km}$. Therefore, there is a relevant discrepancy between the temporal variations of TEC in ONRJ in comparison with temporal variations of TEC in RIOD, as shown in Fig. 7 (right panel). Another issue that affected PPP/tec is shown for day 20 , where it is possible to see that there is low variability in the TEC difference between ONRJ and RIOD. On this day, $\mathrm{PPP} /$ if also showed a low performance, which is explained
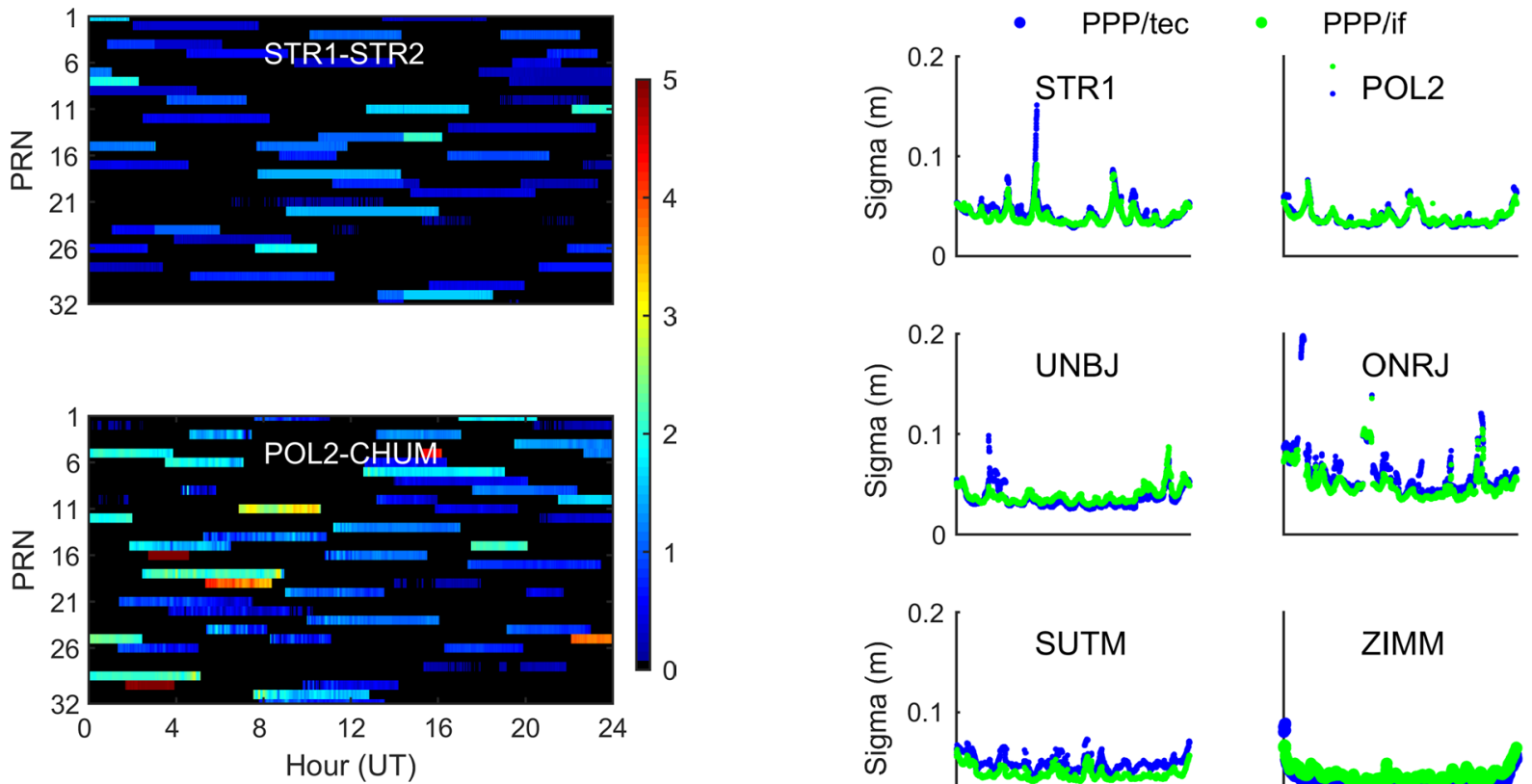

Fig. 6 Standard deviation of the PPP solution for day 46 of 2013 

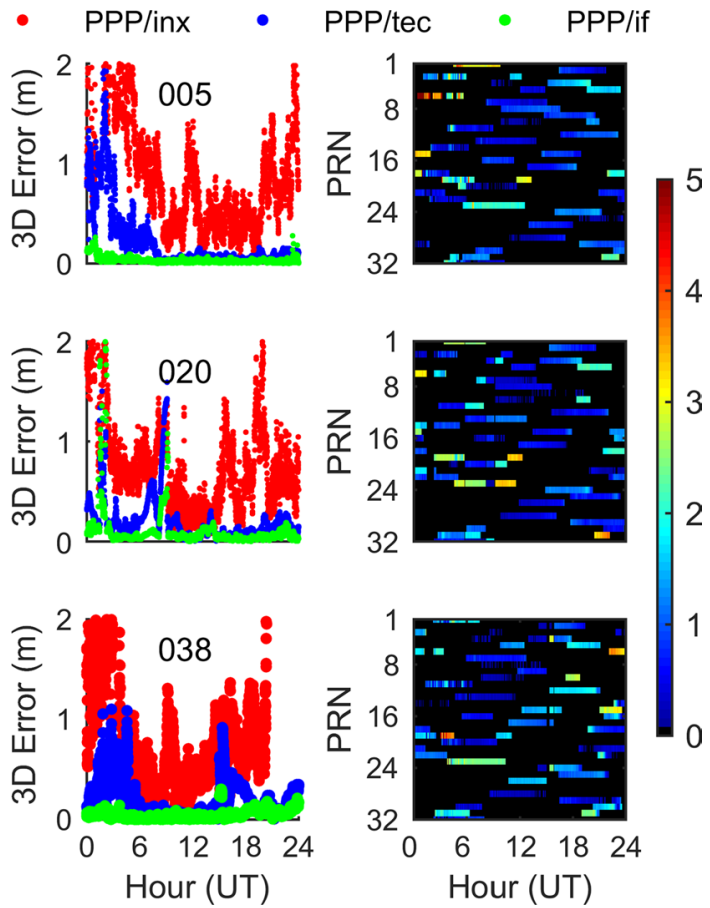

Fig. 7 PPP results from station ONRJ for days 5, 20 and 38 of 2013 (left) and the discrepancies of TEC between ONRJ and RIOD (right). The color bar represents the discrepancy in TECU, where the black color represents the absence of data

by registration failures in the station ONRJ. Therefore, it was not possible to get an accurate result for PPP/tec on day 20 of 2013. Finally, day 38 presented a relevant degradation in the PPP/tec during two periods. The first corresponds to the evening prereversal enhancement period, where there is high variability of TEC difference between the ONRJ and RIOD stations. The second is between 16 and 20 UT when severe data collection failures were detected at the RIOD base station, and therefore, only PPP/tec presented a low performance for station ONRJ.

With the aim of showing an overview of the PPP/tec performance for the days analyzed, Fig. 8 shows the daily 3D RMSE of the solutions. For each day, we calculated a unique value of RMSE considering the errors for each epoch processed in kinematic mode. Days with problems similar to day 20 for ONRJ (registration failures in the rover stations) were excluded from the sample. However, days with problems similar to day 38 for ONRJ (registration failures in base stations) were considered. It is worth mentioning that, although POL2 and CHUM have the greatest distance between base and rover stations, PPP/tec for the POL2 station presented better accuracy than PPP/tec for ONRJ. Such consideration enables us to conclude that the main factor that affected PPP/ tec accuracy in the experiments was the ionospheric variability in the southern crest region of the enhanced TEC associated with the equatorial ionization anomaly.

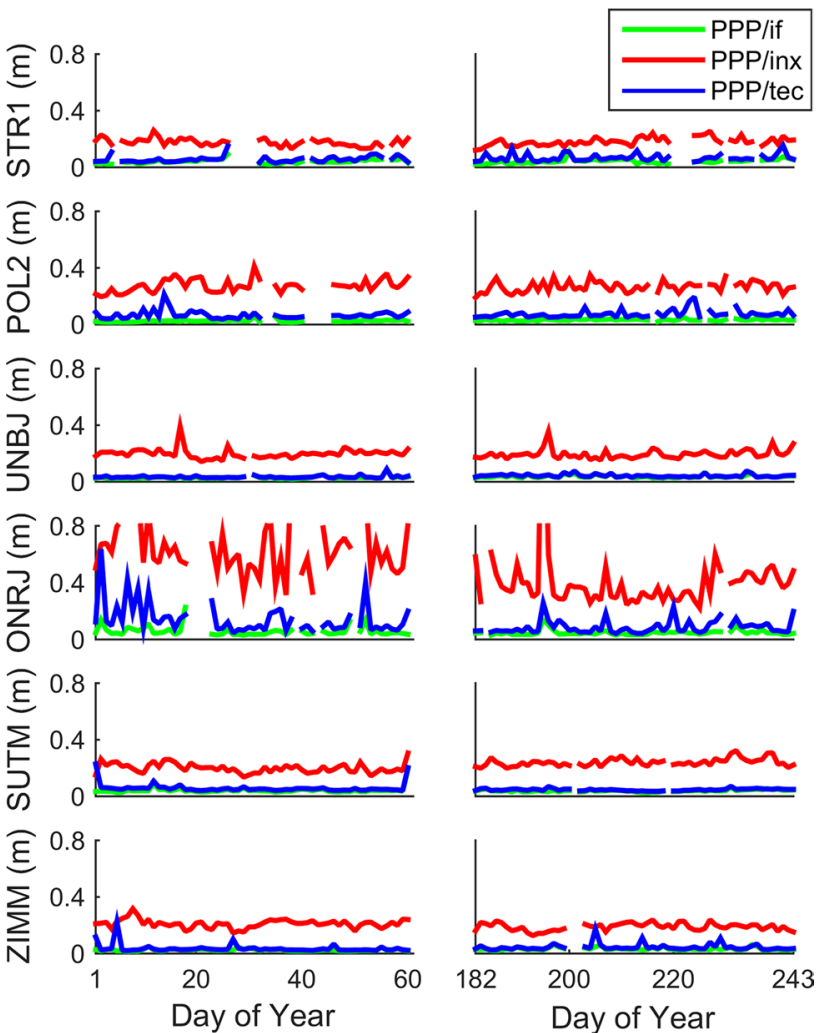

Fig. 8 Temporal series of the 3D RMSE obtained for each day analyzed

Table 3 shows an overview of the PPP results. In general, the RMSE performance of PPP/tec was $74 \%$ better than PPP/ inx, and the total difference of RMSE between PPP/tec and PPP/if was $0.04 \mathrm{~m}$. Regarding the standard deviation, PPP/ tec showed an improvement of $89.5 \%$ when compared with $\mathrm{PPP} / \mathrm{inx}$. Also, similar values in the standard deviation of $\mathrm{PPP} /$ tec and PPP/if were obtained, which show that these solutions were affected by similar random errors. Thus, we can say that the proposed method for TEC estimation enables us to obtain a relevant improvement for PPP in comparison with PPP using IONEX of CODE files. We can also affirm that the PPP/tec solution presented similar accuracy to that of PPP when the first-order ionospheric effect is eliminated, but with a slightly worse performance of 3D RMSE.

\section{Discussion and conclusions}

We have successfully implemented a procedure to estimate TEC in a base station and obtain a single-frequency PPP solution similar to that of dual-frequency PPP solution in nearby rover stations, but with a slightly worse performance of 3D RMSE. We verified that, despite TEC differences between base and rover stations reaching values of 1-5 TECU, these differences did not significantly affect PPP 
Table 3 3D RMSE and standard deviation obtained from all stations and days analyzed

\begin{tabular}{|c|c|c|c|c|c|c|}
\hline \multirow[t]{2}{*}{ Rover stations } & \multicolumn{2}{|c|}{ PPP/if (m) } & \multicolumn{2}{|c|}{ PPP/tec (m) } & \multicolumn{2}{|c|}{ PPP/inx (m) } \\
\hline & RMSE & Sigma & RMSE & Sigma & RMSE & Sigma \\
\hline STR1 & 0.04 & 0.04 & 0.07 & 0.05 & 0.18 & 0.54 \\
\hline POL2 & 0.03 & 0.04 & 0.08 & 0.05 & 0.27 & 0.50 \\
\hline UNBJ & 0.03 & 0.04 & 0.04 & 0.04 & 0.20 & 0.37 \\
\hline ONRJ & 0.06 & 0.09 & 0.14 & 0.10 & 0.58 & 0.76 \\
\hline SUTM & 0.04 & 0.06 & 0.06 & 0.07 & 0.22 & 0.79 \\
\hline ZIMM & 0.03 & 0.04 & 0.05 & 0.04 & 0.20 & 0.29 \\
\hline All & 0.04 & 0.05 & 0.08 & 0.06 & 0.31 & 0.54 \\
\hline
\end{tabular}

accuracy. On the other hand, the PPP solution was substantially affected when we verified problems to represent the temporal variations of TEC in the rover stations, but, in general, the description of temporal TEC variability was good enough for high accuracy in PPP solutions.

In the results of our experiments, although the proposed method provides an improvement of $74 \%$ in comparison with the CODE VTEC maps, the estimated DCBs between the methods were quite similar. Nowadays, DCBs are regarded as one of the main factors limiting the accuracy of ionospheric models. Actually, great uncertainty in the absolute accuracy of TEC remains due to DCBs, which can affect physical analysis of the ionosphere and cause distortion between observations measured by different instruments. The distortion between TEC retrieved by GNSS and parameters measured by different instruments, such as ionosondes and topside sounders, can cause a relevant problem for ionospheric models based on data assimilation methods. However, based on the results shown, the DCB estimation cannot be considered a current problem for PPP applications.

The temporal variations of the ionosphere can already be well estimated. In addition, it is possible to avoid the intrinsic errors of the mapping functions to convert TEC in VTEC by using the slant TEC directly in the GNSS positioning. Therefore, from now on, one can say that the principal challenge of the community developing ionospheric models for GNSS positioning is not DCB or temporal variation of the ionosphere, but the development of accurate spatial interpolation methods for TEC and/or the improvement of mapping functions. The spatial variations of the ionosphere were not addressed as a relevant problem in the TEC assessment because we used short distances between base and rover stations. This procedure cannot be currently applied to global users or in regions with a sparse network of stations. However, further investigations can be carried out for defining modeling processes and/or spatial interpolations to accurately obtain TEC for single-frequency PPP applications in larger areas. As far as spatial interpolation processes are improved, it is possible to conclude from the experimental analysis that the procedures for TEC calibration, with a similar formulation as the one presented, are emerging as having potential for a wide range of applications for those using $\mathrm{L} 1$ receivers.

Acknowledgements This work was jointly funded by Coordenação de Aperfeiçoamento de Pessoal de Nível Superior (CAPES), Fundação de Amparo à Pesquisa do Estado de São Paulo (FAPESP-Grant: 2015/15027-7) and Conselho Nacional de Desenvolvimento Científico e Tecnológico (CNPq Grants: 304674/2014-1 and 429885/2016-4). The authors are grateful to CODE for providing IONEX files, Instituto Brasileiro de Geografia e Estatística (IBGE) and IGS for providing data from dual-frequency GNSS receivers.

\section{References}

Alizadeh MM, Schuh H, Schmidt M (2015) Ray tracing technique for global 3-D modeling of ionospheric electron density using GNSS measurements. Radio Sci 50(6):539-553

Arikan F, Nayir H, Sezen U, Arikan O (2008) Estimation of single station interfrequency receiver bias using GPS-TEC. Radio Sci 43(4):RS4004

Azpilicueta F, Brunini C, Radicella SM (2006) Global ionospheric maps from GPS observations using modip latitude. Adv Space Res 38(11):2324-2331

Biqiang Z, Weixing W, Libo L, Tian M (2007) Morphology in the total electron content under geomagnetic disturbed conditions: results from global ionosphere maps. Ann Geophys 25(7):1555-1568

Brunini C, Meza A, Azpilicueta F, Van Zele MA, Gende M, Díaz A (2004) A new ionosphere monitoring technology based on GPS. Astrophys Space Sci 290(3):415-429

Bust GS, Mitchell CN (2008) History, current state, and future directions of ionospheric imaging. Rev Geophys 46(1):RG1003

Camargo PO, Monico JFG, Ferreira LDD (2000) Application of ionospheric corrections in the equatorial region for L1 GPS users. Earth Planets Space 52(11):1083-1089

Ciraolo L, Azpilicueta F, Brunini C, Meza A, Radicella SM (2007) Calibration errors on experimental slant total electron content (TEC) determined with GPS. J Geod 81(2):111-120

Coco DS, Coker C, Dahlke SR, Clynch JR (1991) Variability of GPS satellite differential group delay biases. IEEE Trans Aerosp Electron Syst 27(6):931-938

Datta-Barua S, Lee J, Pullen S, Luo M, Ene A, Qiu D, Zhang G, Enge $P$ (2010) Ionospheric threat parameterization for local area global-positioning-system-based aircraft landing systems. J Aircr 47(4):1141-1151

Durmaz M, Karslioğlu MO (2015) Regional vertical total electron content (VTEC) modeling together with satellite and receiver differential code biases (DCBs) using semi-parametric 
multivariate adaptive regression B-splines (SP-BMARS). J Geod 89(4):347-360

Elmas ZG, Aquino M, Marques HA, Monico JFG (2011) Higher order ionospheric effects in GNSS positioning in the European region. Ann Geophys 29(8):1383-1399

Feltens J (1998) Chapman profile approach for 3-D global TEC representation, IGS presentation. In: Proceedings of the 1998 IGS analysis centers workshop, ESOC, Darmstadt, Germany, 9-11 Feb 1998, pp 285-297

Georgiadiou Y (1994) Modelling the ionosphere for an active control network of GPS stations. LGR series 7. Delft Geodetic Computing Centre, Delft

Hajj GA, Wilson BD, Wang C, Pi X, Rosen IG (2004) Data assimilation of ground GPS total electron content into a physics-based ionospheric model by use of the Kalman filter. Radio Sci 39:RS1S05

He H, Li J, Yang Y, Xu J, Guo H, Wang A (2014) Performance assessment of single- and dual-frequency BeiDou/GPS single-epoch kinematic positioning. GPS Solut 18(3):393-403

Hernández-Pajares M, Juan JM, Sanz J (1999) New approaches in global ionospheric determination using ground GPS data. J Atmos Solar Terr Phys 61(16):1237-1247

Hernández-Pajares M, Juan JM, Sanz J, Orus R, Garcia-Rigo A, Feltens J, Komjathy A, Schaer SC, Krankowski A (2009) The IGS VTEC maps: a reliable source of ionospheric information since 1998. J Geod 83(3):263-275

Jin SG, Jin R, Li D (2016) Assessment of Beidou differential code bias variations from multi-GNSS network observations. Ann Geophys 34(2):259-269

Le AQ, Tiberius C (2007) Single-frequency precise point positioning with optimal filtering. GPS Solut 11(1):61-69

Li Z, Yuan Y, Li H, Ou J, Huo X (2012) Two-step method for the determination of the differential code biases of COMPASS satellites. J Geod 86(11):1059-1076

Li X, Ge M, Zhang H, Wickert J (2013) A method for improving uncalibrated phase delay estimation and ambiguity-fixing in real-time precise point positioning. J Geod 87(5):405-416

Lin CH, Richmond AD, Liu JY, Yeh HC, Paxton LJ, Lu G, Tsai HF, Su S-Y (2005) Large-scale variations of the low-latitude ionosphere during the October-November 2003 superstorm: observational results. J Geophys Res 110(A9):A09S28

Macalalad EP, Tsai L-C, Wu J (2016) Performance evaluation of different ionospheric models in single-frequency code-based differential GPS positioning. GPS Solut 20(2):173-185

Mannucci AJ, Wilson BD, Yuan DN, Ho CH, Lindqwister UJ, Runge TF (1998) A global mapping technique for GPS-derived ionospheric total electron content measurements. Radio Sci 33(3):565-582

Marques HA, Monico JFG, Aquino M (2011) Rinex HO: second-and third-order ionospheric corrections for RINEX observation files. GPS Solut 15(3):305-314

Migoya-Orué Y, Nava B, Radicella SM, Alazo-Cuartas K (2015) GNSS derived TEC data ingestion into IRI 2012. Adv Space Res 55(8):1994-2002

Mitchell CN, Spencer PSJ (2003) A three-dimensional time-dependent algorithm for ionospheric imaging using GPS. Ann Geophys 46(4):687-696

Montenbruck O, Hauschild A, Steigenberger P (2014) Differential code bias estimation using multi-GNSS observations and global ionosphere maps. J Inst Navig 61(3):191-201

Otsuka Y, Ogawa T, Saito A, Tsugawa T, Fukao S, Miyazaki S (2002) A new technique for mapping of total electron content using GPS network in Japan. Earth Planets Space 54(1):63-70

$\varnothing$-vstedal O (2002) Absolute positioning with single-frequency GPS receivers. GPS Solut 5(4):33-44
Prol FS, Camargo PO (2014) Estimativa da tendência diferencial do código nos receptores GNSS. Bol Ciências Geod 20(4):735-749

Prol FS, Camargo PO (2016) Ionospheric tomography using GNSS: multiplicative algebraic reconstruction technique applied to the area of Brazil. GPS Solut 20(4):807-814

Sardón E, Zarraoa N (1997) Estimation of total electron content using GPS data: how stable are the differential satellite and receiver instrumental biases? Radio Sci 32(5):1899-1910

Schaer S (1999) Mapping and predicting the Earth's ionosphere using the global positioning system. Ph.D. Dissertation, Astronomical Institute, University of Berne, Berne, Switzerland, 25 March

Schunk RW, Scherliess L, Sojka JJ, Thompson DC, Anderson DN, Codrescu M, Minter C, Fuller-Rowell TJ, Heelis RA, Hairston M, Howe BM (2004) Global assimilation of ionospheric measurements (GAIM). Radio Sci 39(1):RS1S02

Sterle O, Stopar B, Prešeren PP (2015) Single-frequency precise point positioning: an analytical approach. J Geod 89(8):793-810

Takasu T, Yasuda A (2009) Development of the low-cost RTK-GPS receiver with an open source program package RTKLIB. In: International symposium on GPS/GNSS, Seogwipo-si Jungmun-dong, Korea, 4-6 Nov

Wen D, Wang Y, Norman R (2012) A new two-step algorithm for ionospheric tomography solution. GPS Solut 16(1):89-94

Wild U (1994) Ionosphere and satellite systems: permanent GPS tracking data for modelling and monitoring. Geodätisch-geophysikalische Arbeiten in der Schweiz, band 48

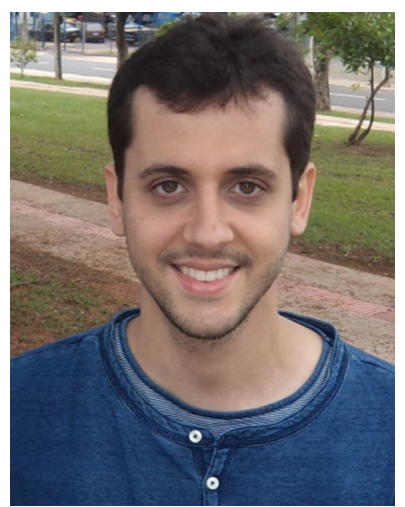

Fabricio dos Santos Prol received his B.E. degree in cartographic engineering in 2011 and M.Sc. degree in cartographic sciences in 2015 from Universidade Estadual Paulista (UNESP) at Presidente Prudente-SP, Brazil. $\mathrm{He}$ is a Ph.D. candidate at UNESP, where the focus of his current research lies in ionospheric modeling using data assimilation.

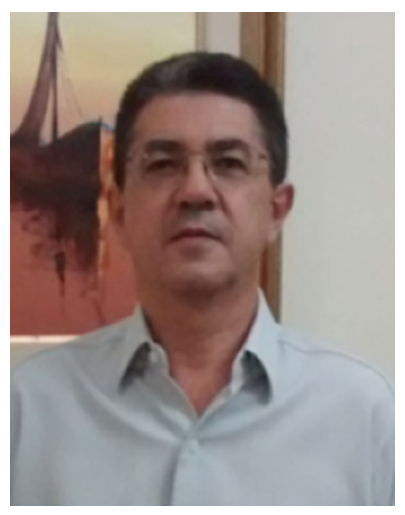

Paulo de Oliveira Camargo received his B.E. degree in cartographic engineering in 1985 from Universidade Estadual Paulista (UNESP), his M.Sc. degree in 1992 and a Ph.D. degree in 1999 in geodetic sciences from the Universidade Federal do Paraná (UFPR), Brazil. He is a professor of the graduate and postgraduate program at UNESP focusing on the following topics: GNSS, ionosphere, quality control and least-squares adjustment. 


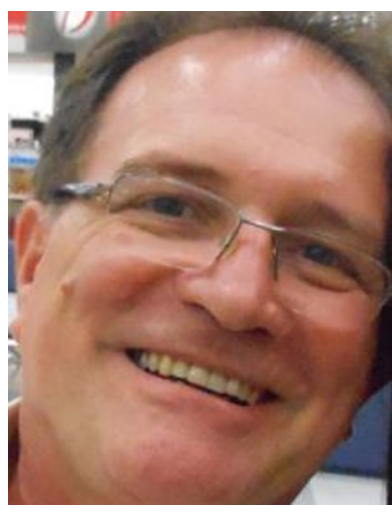

João Francisco Galera Monico received his B.Sc. degree in cartographic engineering in 1982, M.Sc. in geodetic science in 1982 and Ph.D. in space geodesy in 1995. From 2005 to 2013, he was an adjunct professor at the Department of Cartography, São Paulo State University (UNESP) at Presidente Prudente in Brazil, where he was lecturing and researching topics related to GNSS, especially those related to geodesy, surveying and atmospheric sciences. Since September 2013, he is a researcher also with UNESP at the same department.

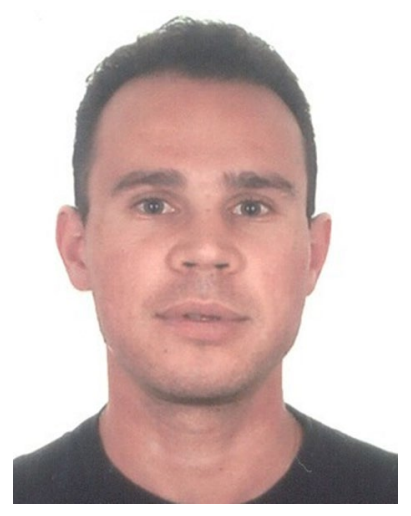

Marcio Tadeu de Assis Honorato Muella is associated professor in the School of Engineering and in the Institute of Research and Development (IP\&D) at the Universidade do Vale do Paraíba (UNIVAP), Brazil. He earned his B.E. degree in electrical and electronic engineering from UNIVAP in 2002, his M.Sc. degree in 2004 and a Ph.D. degree in 2008 in space geophysics from National Institute for Space Research (INPE), Brazil. He works in ionospheric research where the main interest fields of study are the space weather effects on GNSS. 